\title{
OPEN Diffusion kurtosis imaging of gray matter in young adults with autism spectrum disorder
}

\author{
Faye McKenna ${ }^{1,3 凶}$, Laura Miles ${ }^{1}$, Jeffrey Donaldson ${ }^{1}$, F. Xavier Castellanos ${ }^{2,4}$ \& \\ Mariana Lazar ${ }^{1,3}$
}

Prior ex vivo histological postmortem studies of autism spectrum disorder (ASD) have shown gray matter microstructural abnormalities, however, in vivo examination of gray matter microstructure in ASD has remained scarce due to the relative lack of non-invasive methods to assess it. The aim of this work was to evaluate the feasibility of employing diffusional kurtosis imaging (DKI) to describe gray matter abnormalities in ASD in vivo. DKI data were examined for 16 male participants with a diagnosis of ASD and $I Q>80$ and 17 age- and IQ-matched male typically developing (TD) young adults 18-25 years old. Mean (MK), axial (AK), radial (RK) kurtosis and mean diffusivity (MD) metrics were calculated for lobar and sub-lobar regions of interest. Significantly decreased MK, RK, and MD were found in ASD compared to TD participants in the frontal and temporal lobes and several sub-lobar regions previously associated with ASD pathology. In ASD participants, decreased kurtosis in gray matter ROIs correlated with increased repetitive and restricted behaviors and poor social interaction symptoms. Decreased kurtosis in ASD may reflect a pathology associated with a less restrictive microstructural environment such as decreased neuronal density and size, atypically sized cortical columns, or limited dendritic arborizations.

Autism spectrum disorder (ASD) is a neurodevelopmental disorder characterized by a range of social, executive functioning, and sensory deficits ${ }^{1}$, with a prevalence as high as 1 in 50 children $^{2}$. Individuals with ASD have been found to have an array of complex brain abnormalities including early overgrowth ${ }^{3}$, microstructural disorganization $^{4}$, and deficits in both cytoarchitecture ${ }^{5}$ and functional connectivity ${ }^{6}$. In vivo studies of microstructural abnormalities in ASD have focused primarily on white matter ${ }^{7,8}$, leaving the nature and distribution of gray matter (GM) abnormalities largely uncharacterized.

Postmortem studies of ASD have implicated several abnormalities in cortical GM. Early studies found increased cell packing, particularly in the prefrontal cortex $x^{4,9}$ and decreased cell size in the cingulate cortex ${ }^{10}$ and frontal lobe regions ${ }^{4}$, along with heterotopia and decreased dendritic branching in frontal and temporal gyri $^{4,11}$. Ex vivo research has also shown increased markers of inflammation in the anterior cingulate cortex and middle frontal gyrus ${ }^{12}$, along with under-developed minicolumn formation across the cortex ${ }^{5,13-15}$. Although ex vivo studies have been essential for understanding the underlying cellular pathology of ASD, the literature is notably variable likely due to the heterogeneous nature of the disease and the inevitable limitations of postmortem research, which is often based on highly inhomogeneous and small samples ranging from 1 to 11 ASD subjects $^{4,5,10,12-17}$, and typically focuses on isolated brain regions. Thus, techniques for examining GM microstructure in vivo would allow whole-brain analysis within larger, well-matched samples. Such techniques are necessary for better describing the location, degree and developmental course of GM microstructural pathology and their variation across individuals affected with ASD.

Diffusion-weighted MRI (dMRI) is a powerful tool for describing microstructural tissue organization at micrometer scale by probing water molecule displacement, which is sensitive to microscopical barriers (e.g., membranes) and organelle organization (e.g., density, orientation) within each voxel. To date, diffusion tensor imaging (DTI), which approximates water diffusion in tissue using a Gaussian distribution, has remained the most widely used diffusion technique. DTI associated indices, fractional anisotropy (FA), and mean (MD), axial $(\mathrm{AD})$, and radial $(\mathrm{RD})$ diffusivities have been widely applied to characterize anisotropic white matter and have

\footnotetext{
${ }^{1}$ Department of Radiology, Center for Biomedical Imaging, New York University School of Medicine, 660 First Ave, Fourth Floor, New York, NY, USA. ${ }^{2}$ Department of Child and Adolescent Psychiatry, New York University School of Medicine, New York, NY, USA. ${ }^{3}$ Vilcek Institute of Graduate Biomedical Sciences, New York University School of Medicine, New York, NY, USA. ${ }^{4}$ Nathan Kline Institute for Psychiatric Research, Orangeburg, NY, USA. ${ }^{\square}$ email: faye.mckenna@nyulangone.org
} 
been found to be sensitive to a number of white matter pathological changes such as dysmyelination, axonal loss, and inflammation ${ }^{18-21}$. The advent of high angular resolution multi-shell diffusion imaging methods has allowed extending the in vivo investigation of microstructure to gray matter ${ }^{22,23}$. Among these new methods, diffusional kurtosis imaging (DKI) was the first to show high sensitivity to GM changes due to development in typical children and adolescents as well as to document atypical development in attention-deficit hyperactivity disorder (ADHD), a childhood disease ${ }^{24}$. DKI improves upon the DTI characterization of the diffusion signal in tissues by including an additional directional metric, the kurtosis index, to describe non-gaussian diffusion contributions to the MR signal ${ }^{25}$. Non-Gaussian diffusion contributions are inherent to biological tissues such as the brain and arise from the complex organization of microscopic membranes, organelles, and neuronal compartments ${ }^{25}$. Of note, kurtosis metrics have been shown to be able to describe complex structure in the absence of anisotropy ${ }^{25,26}$. Increased kurtosis is assumed to describe increasingly restricted and complex environments and has been shown to characterize brain development and maturation ${ }^{23,24}$, as well as pathological features leading to increased complexity (e.g., astrogliosis or microgliosis) ${ }^{27-29}$. Decreased kurtosis is likely indicative of tissue disintegration and shrinkage ${ }^{30-32}$. DKI provides both a mean kurtosis $(\mathrm{MK})$ metric, which characterizes kurtosis averaged across all spatial directions, and, like DTI, two directional metrics: axial kurtosis (AK), which quantifies kurtosis along the primary or largest diffusivity direction and radial kurtosis (RK), which measures the averaged kurtosis in the plane perpendicular to the primary diffusivity direction. The use of directional kurtosis metrics in GM is supported by recent findings indicating highly directional diffusion in the cortex aligning either radially or both radially and tangentially to the cortical ribbon ${ }^{22,33}$.

To date, DKI has been shown to be sensitive to GM microstructural change in the aging brain ${ }^{34-36}$ and in several conditions including Parkinson's disease ${ }^{37}$, chronic pain ${ }^{38}$, multiple sclerosis ${ }^{39}$, traumatic brain injury ${ }^{40}$, multiple sclerosis ${ }^{39}$, schizophrenia ${ }^{41}$, Alzheimer's disease ${ }^{42}$ and attention-deficit/hyperactivity disorder ${ }^{43}$. Ex vivo studies investigating GM changes in several rodent neurodegenerative models have documented that increased kurtosis parallels increased fiber dispersion ${ }^{29}$, protein accumulation ${ }^{27}$, and astrogliosis ${ }^{28}$, and that decreased kurtosis co-occurs with cytotoxic edema ${ }^{44}$, abnormal neurofilaments, and axonal shrinkage and loss ${ }^{31,45}$. In addition, directional kurtosis estimates have been found to detect unique characteristics of tissue compared to MK in both healthy and clinical samples ${ }^{35,40,46}$. Therefore, we hypothesized that mean and directional kurtosis in the GM of ASD patients is altered as a result of one or more cellular pathological processes observed in ASD: disorganization and alterations of cortical laminar cytoarchitecture and dendrites, decreased cell size, increased cell packing, gliosis, and narrow minicolumn formation ${ }^{4,13,17,47}$.

To date, only few studies have investigated GM cortical microstructure in ASD using in vivo dMRI methods ${ }^{48-50}$. An initial study found slightly increased MD in total brain GM in adolescents with ASD ages 12-18 years old ${ }^{48}$, while another study employed a Restriction Spectrum Imaging (RSI) approach and reported significantly reduced gray matter neurite density (ND) in the left fusiform cortex of adolescents with ASD ages 7-17 years old at multiple-comparison correction level ${ }^{49}$. This second study additionally found slightly increased MD in the parietal and occipital lobes, but this finding did not survive multiple-comparison correction ${ }^{49}$. Most recently, reduced orientation dispersion (ODI) and ND were found in ASD patients with impaired recognition of facial emotional expressions in the occipital lobe, fusiform gyrus, and inferior parietal and superior temporal regions using the Neurite Orientation and Dispersion Imaging (NODDI) approach ${ }^{50}$. Reduced ND in ASD was hypothesized to be related to the ex vivo finding of a lower density of, or thinning of, myelinated axons in the gray matter cortex, while decreased ODI was postulated to be associated with the loss of dendritic spines ${ }^{48,49}$. The increased MD noted in two of the studies was proposed to be a result of increased water due to the inflammatory response often reported in $\mathrm{ASD}^{48,49}$. This previous work has suggested that dMRI may be able to distinguish different GM microstructural pathologies in ASD.

In this study, we employed DKI to characterize, non-invasively and in vivo, gray matter cortical differences in a group of young adults with ASD versus age, sex and IQ-matched typically developing (TD) control participants and to test the hypothesis that kurtosis is associated with the severity of core ASD symptom deficits (i.e., communication, repetitive behaviors, and social interaction). Compared to biological model-based multi-shell dMRI methods, such as NODDI, DKI is a data driven mathematical model that makes no assumptions regarding underlying microstructure, and thus is independent of such assumptions, which may not be valid in various pathologies. Moreover, compared to DTI metrics, DKI metrics have been shown to be robust with respect to partial volume averaging with cerebro-spinal fluid ${ }^{51}$, an artifact likely to at least partially affect cortical regions.

Results

Participants. Demographic characteristics for both groups are presented in Table 1. There were no group differences in age $(p=0.678)$, education $(p=0.463)$, or full-scale IQ score $(p=0.143)$ (Table 1$)$.

Between-group comparisons. At the lobar level, individuals with ASD demonstrated significantly lower $\mathrm{RK}$ in the left frontal, and right and left temporal lobe GM, and significantly lower MK in the right temporal lobe $\mathrm{GM}$ at the $\mathrm{q} \leq 0.05 \mathrm{BH}$ FDR-corrected cut-off ${ }^{52}$ (Table 2; Fig. 1).

At the sub-lobar level, RK demonstrated the most robust between-group differences with a significant decrease in the ASD group noted in the left pars opercularis division of the inferior frontal gyrus, left superior frontal and bilateral superior temporal gyri, right lateral orbital frontal, bilateral precuneus and left insular cortex regions of interest (ROIs) (Table 2; Fig. 2). Significantly decreased MK in ASD was found in the bilateral pars opercularis division of the inferior frontal gyrus, left superior temporal gyri, right lateral orbital frontal, right precuneus and left insular cortices ROIs (Table 2; Fig. 2). Between-group MD comparisons revealed fewer yet similar regions of decreased diffusivity in ASD compared to TD participants shown through analyses of RK and MK with only the right posterior division of the cingulate cortex reaching significance after multiple comparison corrections. 


\begin{tabular}{|c|c|c|c|}
\hline & Autism spectrum disorder $(n=16)$ & Typically developing $(n=17)$ & t-test $p$ value \\
\hline Male/female & $16 / 0$ & $17 / 0$ & - \\
\hline Handedness (right/left) & $15 / 1$ & $16 / 1$ & .809 \\
\hline Age (years) Mean \pm SD & $21.4 \pm 2.4$ & $21.7 \pm 2.1$ & .678 \\
\hline Range & $18-25$ & $18-24$ & \\
\hline Education (years) Mean \pm SD & $14.90 \pm 1.70$ & $15.3 \pm 1.5$ & .463 \\
\hline Range & $12-18$ & $13-17$ & \\
\hline WAIS-III FSIQ Mean \pm SD & $108.88 \pm 17.39$ & $116.65 \pm 11.98$ & .143 \\
\hline Range & $85-148$ & $98-143$ & \\
\hline ADI-R RRB Mean \pm SD & $5.55 \pm 1.75$ & - & - \\
\hline Range & $3-9$ & & \\
\hline ADI-R COM Mean \pm SD & $15.27 \pm 5.0$ & - & - \\
\hline Range & $9-24$ & & \\
\hline ADI-R SOC Mean \pm SD & $19.18 \pm 7.0$ & - & - \\
\hline Range & $6-30$ & & \\
\hline ADOS SOC Mean \pm SD & $3.88 \pm 1.6$ & - & - \\
\hline Range & $1-6$ & & \\
\hline $\mathrm{ADOS}$ COM Mean $\pm \mathrm{SD}$ & $1.38 \pm 0.7$ & - & - \\
\hline Range & $1-3$ & & \\
\hline ADOS COM SOC Mean \pm SD & $3.88 \pm 1.5$ & - & - \\
\hline Range & $1-6$ & & \\
\hline ADOS SARRB Mean \pm SD & $5.31 \pm 2.1$ & - & - \\
\hline Range & $1-7$ & & \\
\hline ADOS SBRI Mean \pm SD & $1.06 \pm 0.3$ & - & - \\
\hline Range & $1-2$ & & \\
\hline ADOS SA Mean \pm SD & $4.31 \pm 2.2$ & - & - \\
\hline Range & $1-8$ & & \\
\hline
\end{tabular}

Table 1. Summary of demographic, IQ, ADIR, and ADOS severity scores of the participants in TD and ASD groups. ADIR Autism Diagnostic Interview-Revised, ADOS Autism Diagnostic Observation Schedule, FSIQ Full scale intelligence quotient, $R R B$ Restrictive \& repetitive behaviors, COM Communication, SOC Social Interaction, COM SOC Communication + Social Interaction, SARRB Social Affect \& Restricted Repetitive Behaviors, $S A$ social affect, SBRI Stereotyped behaviors and restricted interests.

No differences in AK were found between groups in either lobar or sub-lobar regions.

Association with disease severity. In the ASD group, increased MK was associated with fewer repetitive and restrictive behaviors and better social interaction, with only the left entorhinal cortex's association with repetitive and restrictive behaviors and the right parietal lobe's association with social interaction reaching significance after correction for multiple comparisons (Table 3; Fig. 3). At trend-level and to a lesser extent, increased RK and MD were correlated to less restrictive and repetitive behaviors and increased RK and MD were correlated to better communication (Supplementary Tables 1 and 2).

\section{Discussion}

The data presented here provides, for the first time, in vivo evidence of GM microstructural pathology in ASD by employing DKI to non-invasively probe tissue organization and complexity. Significantly decreased cortical kurtosis and diffusion noted in the ASD group may reflect one or several pathological processes reported by previous histological studies. The majority of ex vivo studies on ASD found decreased cell size and limited dendritic $\operatorname{arbors}^{17}$, while narrower cell minicolumns with decreased neuropil in the GM of patients with ASD have been extensively documented ${ }^{5,13,16}$. These findings suggest reduced microstructural complexity in the GM of the ASD brain, consistent with our findings of decreased MK and RK. Further supporting this hypothesis, rodent studies have reported that decreases in MK may reflect neuronal loss, microgliosis, myelin disruption ${ }^{31}$, neuron disorganization and cytotoxic edema ${ }^{44}$, with decreases in both MK and RK reflecting reduced GM neurite density ${ }^{53}$.

$\mathrm{MK}, \mathrm{RK}$, and MD measure different characteristics of water diffusion, and are likely sensitive to somewhat different microstructural abnormalities in GM tissue ${ }^{25}$. In the data investigated here, RK, a DKI-specific parameter of diffusional kurtosis perpendicular to the primary direction of diffusion, was the most sensitive metric in quantifying between-group differences, highlighting areas with known cellular pathology in ASD (Table 2; Figs. 2 and 4). The decreased MK in ASD was primarily driven by decreases in RK, with AK showing no quantifiable between-group differences. MD, a classic measure of Gaussian diffusion, was less sensitive in detecting GM pathology in ASD, therefore highlighting non-Gaussian kurtosis metrics as more powerful in detecting GM microstructural changes. 


\begin{tabular}{|c|c|c|c|c|c|c|c|c|c|c|c|c|c|}
\hline Region & $R / L$ & MK ASD & MK TD & $\begin{array}{l}\text { ANCOVA } p \\
\text { values MK }\end{array}$ & $\begin{array}{l}\text { MK } \\
\text { Cohen's d }\end{array}$ & RK ASD & RK TD & $\begin{array}{l}\text { ANCOVA } p \\
\text { values RK }\end{array}$ & $\begin{array}{l}\text { RK Cohen's } \\
\text { d }\end{array}$ & $\begin{array}{l}\text { MD ASD } \\
\left(\mu \mathrm{m}^{2} / \mathrm{ms}\right)\end{array}$ & $\begin{array}{l}\text { MD TD } \\
\left(\mu \mathrm{m}^{2} / \mathrm{ms}\right)\end{array}$ & $\begin{array}{l}\text { ANCOVA } p \\
\text { values MD }\end{array}$ & $\begin{array}{l}\text { MD } \\
\text { Cohen's } \\
\text { d }\end{array}$ \\
\hline \multicolumn{14}{|c|}{ Frontal lobe } \\
\hline Frontal lobe & Right & $.70 \pm .02$ & $.72 \pm .03$ & .039 & .232 & $.71 \pm .03$ & $.73 \pm .03$ & .028 & .395 & $1.18 \pm .09$ & $1.25 \pm .07$ & .030 & .408 \\
\hline Frontal lobe & Left & $.71 \pm .02$ & $.72 \pm .03$ & .034 & .271 & $.71 \pm .02$ & $.74 \pm .03$ & $.011^{*}$ & .462 & - & - & - & - \\
\hline $\begin{array}{l}\text { Caudal mid- } \\
\text { dle frontal }\end{array}$ & Left & $.73 \pm .03$ & $.76 \pm .04$ & .038 & .391 & $.74 \pm .03$ & $.79 \pm .05$ & .020 & .499 & - & - & - & - \\
\hline \begin{tabular}{l|} 
Lateral \\
orbitofrontal
\end{tabular} & Right & $.66 \pm .04$ & $.68 \pm .03$ & $.001^{*}$ & .418 & $.63 \pm .05$ & $.67 \pm .04$ & $.002^{*}$ & .447 & - & - & - & - \\
\hline $\begin{array}{l}\text { Lateral } \\
\text { orbitofrontal }\end{array}$ & Left & $.67 \pm .03$ & $.68 \pm .03$ & .010 & .179 & $.64 \pm .04$ & $.66 \pm .04$ & .011 & .205 & - & - & - & - \\
\hline Paracentral & Right & $.71 \pm .05$ & $.72 \pm .05$ & .022 & .031 & - & - & - & - & $1.21 \pm .13$ & $1.31 \pm .11$ & .029 & .409 \\
\hline $\begin{array}{l}\text { Pars opercu- } \\
\text { laris }\end{array}$ & Right & $.71 \pm .03$ & $.72 \pm .03$ & $.004^{\star}$ & .302 & $.72 \pm .03$ & $.75 \pm .04$ & .014 & .432 & - & - & - & - \\
\hline $\begin{array}{l}\text { Pars opercu- } \\
\text { laris }\end{array}$ & Left & $.71 \pm .03$ & $.73 \pm .03$ & $.004^{*}$ & .398 & $.72 \pm .03$ & $.76 \pm .03$ & $<.001^{\star}$ & .561 & - & - & - & - \\
\hline $\begin{array}{l}\text { Pars trian- } \\
\text { gularis }\end{array}$ & Right & $.73 \pm .31$ & $.74 \pm .04$ & .025 & .144 & $.73 \pm .04$ & $.76 \pm .05$ & .034 & .309 & $1.14 \pm .09$ & $1.19 \pm .05$ & .047 & .381 \\
\hline Precentral & Left & - & - & - & - & - & - & - & - & $1.25 \pm .09$ & $1.32 \pm .10$ & .050 & .372 \\
\hline $\begin{array}{l}\text { Rostral mid- } \\
\text { dle frontal }\end{array}$ & Left & - & - & - & - & $.71 \pm .02$ & $.73 \pm .04$ & .017 & .425 & - & - & - & - \\
\hline $\begin{array}{l}\text { Superior } \\
\text { frontal }\end{array}$ & Right & - & - & - & - & $.68 \pm .03$ & $.70 \pm .05$ & .022 & .253 & $1.19 \pm .09$ & $1.28 \pm .09$ & .007 & .516 \\
\hline $\begin{array}{l}\text { Superior } \\
\text { frontal }\end{array}$ & Left & $.69 \pm .03$ & $.71 \pm .03$ & .035 & .251 & $.69 \pm .04$ & $.72 \pm .04$ & $.002^{*}$ & .412 & - & - & - & - \\
\hline \multicolumn{14}{|l|}{ Parietal lobe } \\
\hline Parietal lobe & Right & - & - & - & - & - & - & - & - & $1.20 \pm .07$ & $1.27 \pm .1$ & .039 & .395 \\
\hline Parietal lobe & Left & - & - & - & - & $.74 \pm .03$ & $76 \pm .03$ & .039 & .346 & - & - & - & \\
\hline $\begin{array}{l}\text { Inferior } \\
\text { parietal }\end{array}$ & Right & - & - & - & - & $.74 \pm .03$ & $.76 \pm .03$ & .047 & .287 & - & - & - & - \\
\hline Precuneus & Right & $.68 \pm .04$ & $.70 \pm .03$ & $.003^{*}$ & .327 & $.69 \pm .05$ & $.72 \pm .04$ & $.005^{*}$ & .307 & - & - & - & - \\
\hline Precuneus & Left & $.68 \pm .03$ & $.71 \pm .03$ & .029 & .330 & $.69 \pm .04$ & $.72 \pm .03$ & $.001^{*}$ & .441 & - & - & - & - \\
\hline $\begin{array}{l}\text { Superior } \\
\text { parietal }\end{array}$ & Right & - & - & - & - & - & - & - & - & $1.3 \pm .10$ & $1.4 \pm .15$ & .022 & .442 \\
\hline $\begin{array}{l}\text { Supramar- } \\
\text { ginal }\end{array}$ & Right & - & - & - & - & $.72 \pm .04$ & $.75 \pm .03$ & .023 & .454 & - & - & - & - \\
\hline \multicolumn{14}{|c|}{ Temporal lobe } \\
\hline $\begin{array}{l}\text { Temporal } \\
\text { lobe }\end{array}$ & Right & $.67 \pm .03$ & $.70 \pm .02$ & $.005^{*}$ & .547 & $.64 \pm .04$ & $.69 \pm .03$ & $<.001^{\star}$ & .694 & - & - & - & - \\
\hline $\begin{array}{l}\text { Temporal } \\
\text { lobe }\end{array}$ & Left & - & - & - & - & $.64 \pm .04$ & $.68 \pm .03$ & $.002^{*}$ & .622 & $1.09 \pm .05$ & $1.12 \pm .04$ & .040 & .280 \\
\hline Bankssts & Right & $.72 \pm .03$ & $.75 \pm .04$ & .031 & .443 & $.73 \pm .04$ & $.77 \pm .05$ & .017 & .452 & - & - & - & - \\
\hline Fusiform & Right & - & - & - & - & $.63 \pm .06$ & $.67 \pm .04$ & .018 & .460 & - & - & - & - \\
\hline $\begin{array}{l}\text { Parahip- } \\
\text { pocampal }\end{array}$ & Right & - & - & - & - & $.62 \pm .07$ & $.66 \pm .04$ & .021 & .344 & - & - & - & - \\
\hline $\begin{array}{l}\text { Parahip- } \\
\text { pocampal }\end{array}$ & Left & - & - & - & - & $.59 \pm .06$ & $.65 \pm .05$ & .024 & .477 & - & - & - & - \\
\hline $\begin{array}{l}\text { Inferior } \\
\text { temporal }\end{array}$ & Right & - & - & - & - & $.58 \pm .04$ & $.63 \pm .05$ & .015 & .552 & - & - & - & - \\
\hline $\begin{array}{l}\text { Middle } \\
\text { temporal }\end{array}$ & Right & - & - & - & - & $.64 \pm .04$ & $.68 \pm .04$ & .019 & .503 & $1.02 \pm .04$ & $1.06 \pm .06$ & .042 & .386 \\
\hline $\begin{array}{l}\text { Middle } \\
\text { temporal }\end{array}$ & Left & $.65 \pm .04$ & $.69 \pm .03$ & .027 & .509 & $.63 \pm .04$ & $.67 \pm .04$ & .011 & .572 & - & - & - & - \\
\hline $\begin{array}{l}\text { Superior } \\
\text { temporal }\end{array}$ & Right & $.70 \pm .03$ & $.72 \pm .03$ & .015 & .486 & $.69 \pm .03$ & $.73 \pm .03$ & $.001^{*}$ & .660 & $1.14 \pm .06$ & $1.23 \pm .12$ & .008 & .531 \\
\hline $\begin{array}{l}\text { Superior } \\
\text { temporal }\end{array}$ & Left & $.69 \pm .03$ & $.72 \pm .02$ & $.004^{*}$ & .637 & $.68 \pm .03$ & $.72 \pm .03$ & $.003^{*}$ & .663 & - & - & - & - \\
\hline $\begin{array}{l}\text { Temporal } \\
\text { pole }\end{array}$ & Right & - & - & - & - & - & - & - & - & $1.16 \pm .14$ & $1.30 \pm .15$ & .014 & .457 \\
\hline \multicolumn{14}{|l|}{ Other } \\
\hline Insula & Right & $.66 \pm .04$ & $.68 \pm .03$ & .049 & .374 & & & - & - & - & - & - & - \\
\hline Insula & Left & $.65 \pm .03$ & $.68 \pm .03$ & $.003^{*}$ & .492 & $.67 \pm .03$ & $.71 \pm .04$ & $.002^{*}$ & .528 & - & - & - & - \\
\hline $\begin{array}{l}\text { Isthmus } \\
\text { cingulate }\end{array}$ & Right & $.64 \pm .06$ & $.67 \pm .06$ & .014 & .224 & $.65 \pm .08$ & $.68 \pm .08$ & .035 & .160 & - & - & - & - \\
\hline $\begin{array}{l}\text { Isthmus } \\
\text { cingulate }\end{array}$ & Left & $.65 \pm .60$ & $.68 \pm .05$ & .015 & .348 & $.66 \pm .08$ & $.69 \pm .06$ & .040 & .275 & - & - & - & - \\
\hline
\end{tabular}




\begin{tabular}{|c|c|c|c|c|c|c|c|c|c|c|c|c|c|}
\hline Region & $\mathrm{R} / \mathrm{L}$ & MK ASD & MK TD & $\begin{array}{l}\text { ANCOVA } p \\
\text { values MK }\end{array}$ & $\begin{array}{l}\text { MK } \\
\text { Cohen's d }\end{array}$ & RK ASD & RK TD & $\begin{array}{l}\text { ANCOVA } p \\
\text { values RK }\end{array}$ & $\begin{array}{l}\text { RK Cohen's } \\
\text { d }\end{array}$ & $\begin{array}{l}\text { MD ASD } \\
\left(\mu \mathrm{m}^{2} / \mathrm{ms}\right)\end{array}$ & $\begin{array}{l}\text { MD TD } \\
\left(\mu \mathrm{m}^{2} / \mathrm{ms}\right)\end{array}$ & $\begin{array}{l}\text { ANCOVA } p \\
\text { values MD }\end{array}$ & $\begin{array}{l}\text { MD } \\
\text { Cohen's } \\
\text { d }\end{array}$ \\
\hline $\begin{array}{l}\text { Caudal } \\
\text { anterior } \\
\text { cingulate }\end{array}$ & Right & - & - & - & - & - & - & - & - & $1.06 \pm .07$ & $1.12 \pm .10$ & .046 & .378 \\
\hline $\begin{array}{l}\text { Posterior } \\
\text { cingulate }\end{array}$ & Right & $.63 \pm .05$ & $.67 \pm .05$ & .009 & .421 & $.64 \pm .07$ & $.69 \pm .08$ & .029 & .317 & $1.03 \pm .07$ & $1.12 \pm .07$ & $.001^{*}$ & .644 \\
\hline $\begin{array}{l}\text { Posterior } \\
\text { cingulate }\end{array}$ & Left & $.65 \pm .04$ & $.69 \pm .06$ & .007 & .338 & $.67 \pm .06$ & $.71 \pm .06$ & .013 & .347 & $1.07 \pm .08$ & $1.13 \pm .09$ & .046 & .358 \\
\hline $\begin{array}{l}\text { Rostral } \\
\text { anterior } \\
\text { cingulate }\end{array}$ & Right & $.59 \pm .04$ & $.62 \pm .05$ & .044 & .404 & - & - & - & - & - & - & - & - \\
\hline $\begin{array}{l}\text { Rostral } \\
\text { anterior } \\
\text { cingulate }\end{array}$ & Left & $.60 \pm .04$ & $.63 \pm .05$ & .013 & .268 & $.60 \pm .04$ & $.63 \pm .06$ & .028 & .287 & - & - & - & - \\
\hline
\end{tabular}

Table 2. Differences in diffusion metrics (MK, RK and $M D)$ in autism spectrum disorder compared to typical developing young adults in the frontal, temporal and parietal lobes and sub-lobar gray matter regions-ofinterest in the brain revealed through ANCOVA analysis controlling for age. All tests shown reach trendlevel at $p \leq 0.05$, uncorrected; tests that remain significant after the $\mathrm{BH}$ procedure for multiple comparisons correction at $\mathrm{q} \leq 0.05$ are indicated by an asterisk ${ }^{*}$ ) along with the effect size (Cohen's $\mathrm{d}$ ).

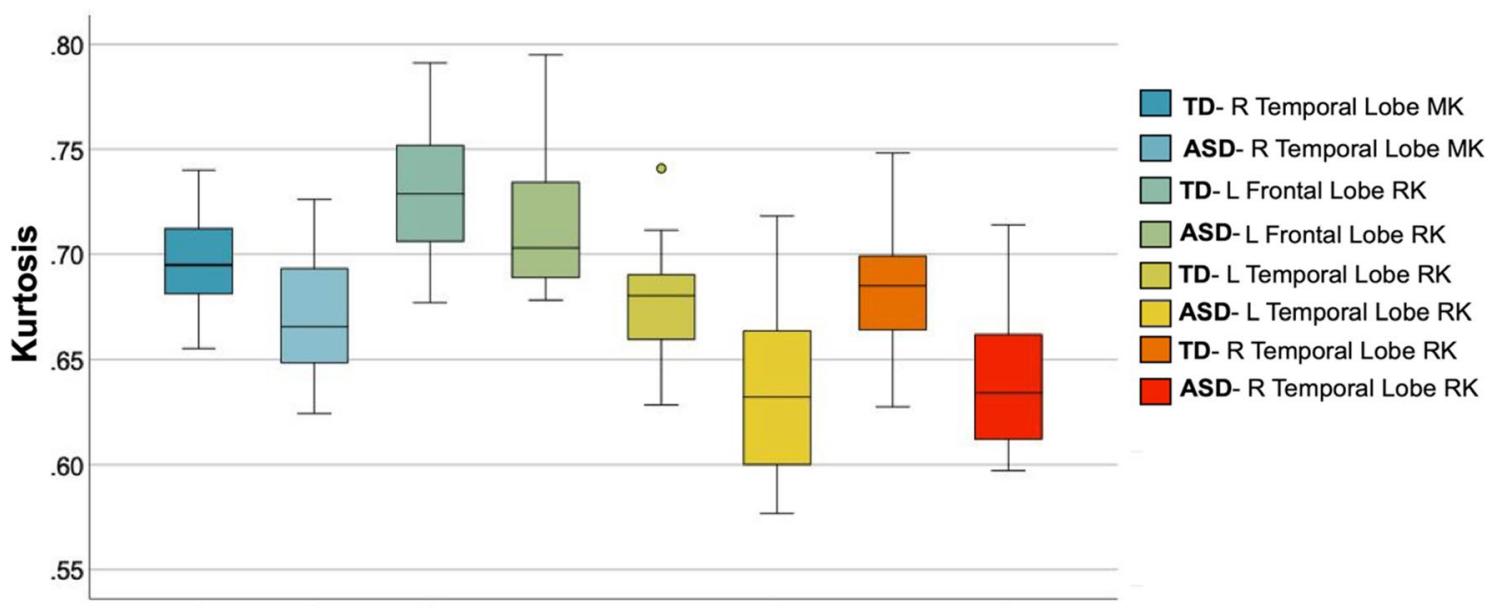

Figure 1. Decreased MK and RK in the right and left temporal lobe GM, and decreased RK in the left frontal lobe GM are observed in autism spectrum disorder compared to the typically developing group. All results shown were significant after ANCOVA between-group tests controlling for age and correcting for multiple comparisons ( $\mathrm{q} \leq .05 \mathrm{BH}$ FDR).

Overall, our data suggest that microstructural changes in ASD are directional and are likely due to alterations in tissue and/or cellular processes affecting diffusion in the plane orthogonal to the primary diffusion direction. Several studies have found that diffusion tensor orientation in GM is closely related to the orientation of neuronal components such as dendritic and axonal fascicles and glial processes ${ }^{5-56}$. Examination of our data (Fig. 5) as well as previous literature shows that the primary direction of diffusion in the cortical mantle, particularly in gyral regions, is perpendicular to cortical mantle ${ }^{33,57-59}$.

This would suggest that radial kurtosis reflects diffusion changes occurring tangential to the cortical mantle. A noteworthy parallel to our finding is the histological documentation of narrower minicolumns with reduced neuropil, which is comprised of dendrites and ascending/descending axons, in frontal and temporal GM regions in patients with $\mathrm{ASD}^{5,15}$. These microscopic columns are aligned perpendicular to the cortical mantle, with narrower unit cytoarchitecture and reduced dendritic processes hypothetically creating less restrictive diffusion tangential to the cortical mantle. Consistent with this hypothesis, our data shows decreased radial kurtosis, i.e., decreased kurtosis perpendicular to minicolumns' direction. In summary, although multiple cellular processes likely contribute in varying degrees to the direction and restriction of diffusion, our findings of reduced radial kurtosis may be driven by the abnormal minicolumn formation previously documented in histological studies.

Compared with the recent dMRI work investigating GM cortical microstructure in ASD using other methods than DKI ${ }^{48-50}$, our findings of significantly decreased kurtosis in the bilateral temporal lobes, precuneus and superior temporal regions generally align with reported findings of decreased neurite density in temporal and parietal lobe ROIs. Decreased DKI was found to be related to decreased neurite density in a preclinical model ${ }^{53}$. In this study, the DKI approach additionally suggests reduced complexity in the frontal lobe and the insular and cingulate cortex areas. More work will be necessary to explore how different dMRI models (e.g. DKI, NODDI, and RSI) compare in identifying GM microstructural pathology in ASD across the lifespan. The slightly increased 


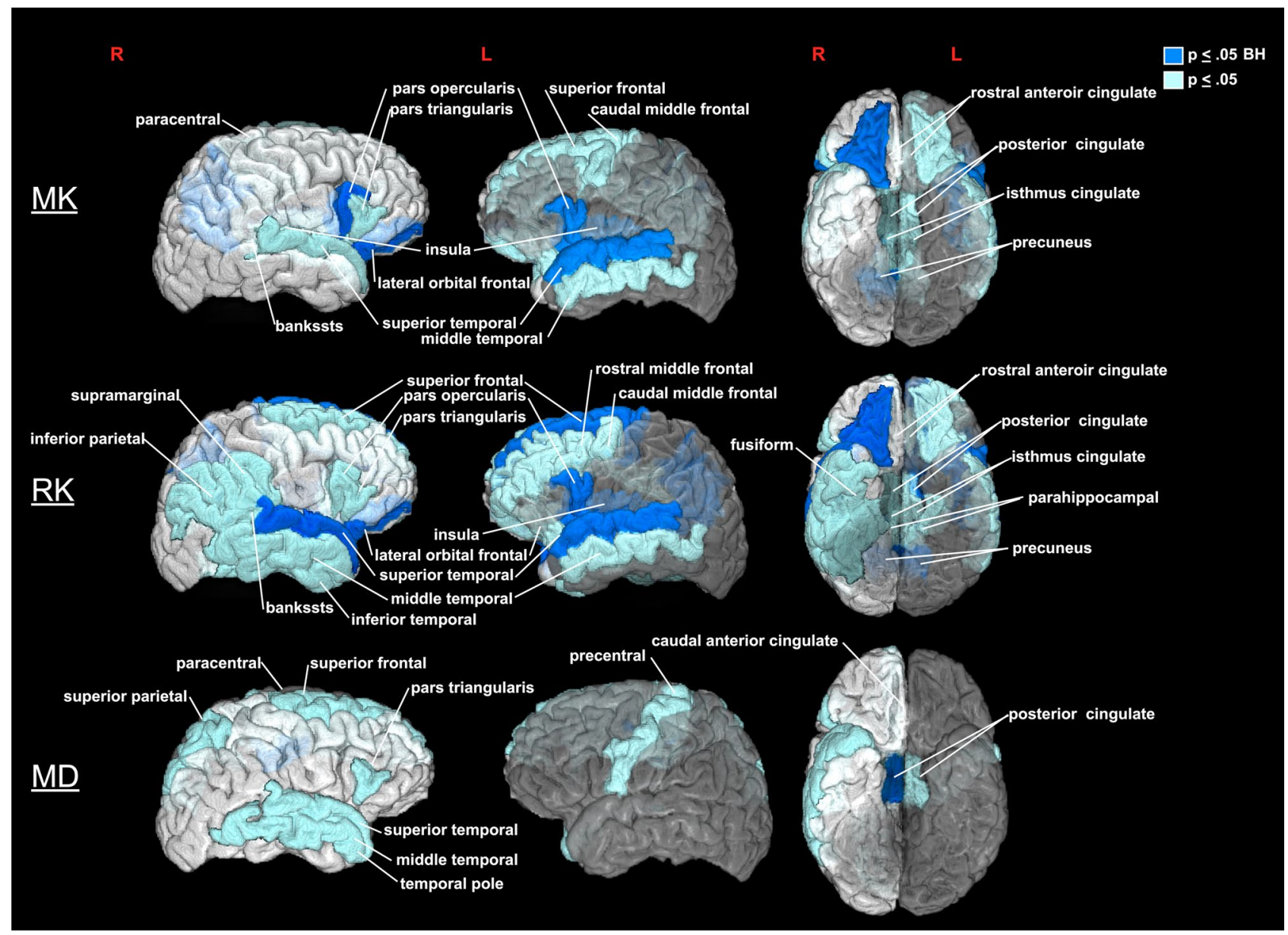

Figure 2. Brain areas with decreased diffusion metrics (MK, RK and $M D)$ in young males with autism spectrum disorder compared to a typically developing group. The color reflects the significance level $(p \leq .05$, uncorrected (light blue) and $\mathrm{q} \leq .05 \mathrm{BH}$ corrected (dark blue). Solid colors are used for outer brain surface areas with see-through regions depicted by transparent colors.

MD values in total brain GM and in the parietal and occipital lobes reported by these previous studies, which however did not pass multiple corrections level, are somewhat contradictory to our findings of significantly decreased MD in the right posterior cingulate cortex ${ }^{48,49}$. More research will be important to elucidate where and to what extend MD is altered in varying ASD populations.

The regions found here to have abnormal microstructure in the ASD group are consistent with both neuroimaging and postmortem neuropathology studies, which highlighted the limbic, frontal and temporal cortical regions as being primarily affected by the disorder ${ }^{12,17,60-65}$ (Table 2; Figs. 1 and 2). Altogether, neuroimaging and cognition studies have suggested that higher-order regions are the most affected cortical regions in ASD, possibly because they are slower maturing and therefore have a longer window of vulnerability to pathological disruptions ${ }^{47}$. Additionally, areas involved in social cognition, such as the inferior frontal gyri, and orbitofrontal, medial prefrontal, posterior parietal, cingulate, superior temporal and insular cortices ${ }^{65,66}$, have been key areas of study in ASD due to their role in social interaction and communication deficits, which are among the disorder's core features ${ }^{67}$. Here we show significant microstructural abnormalities in ASD compared to TD in the pars opercularis division of the inferior frontal and superior frontal gyri, lateral orbital frontal, superior temporal, precuneus, insular and posterior cingulate cortices (Table 2; Fig. 2). Disrupted GM cytoarchitecture of regions within the social-emotional brain network may underlie functional hypoactivation noted using fMRI in an array of tasks including face processing ${ }^{68}$, theory of $\operatorname{mind}^{69}$, motion ${ }^{70}$ and self-referential empathy ${ }^{71}$. Thus, DKI mapping of microstructural pathology in these areas, alongside ex vivo and cognition studies, may better explain the core ASD symptoms of social-emotional impairment that originate from these regions ${ }^{65}$.

Reduced symptom severity in the restrictive and repetitive behaviors subcategory of the Autism Diagnostic Interview-Revised (ADI-R) ${ }^{72}$ was significantly correlated with increased MK in the entorhinal cortex, and reduced symptom severity in the social interaction subcategory of the ADI-R was significantly correlated with increased MK in the right parietal lobe within the ASD group (Table 3; Fig. 3). Increased kurtosis in GM and its associated cellular processes may have a protective function or supporting effect, with increased MK in GM correlating to better performance on executive function tests in traumatic brain injury, multiple sclerosis and 


\begin{tabular}{|c|c|c|c|c|c|}
\hline MK ROI & Hemisphere & Test & $\begin{array}{l}\text { ADI-R restrictive } \\
\text { \&repetitive behaviors } \\
\text { r/p }\end{array}$ & $\begin{array}{l}\text { ADI- } R \text { communication } \\
\mathbf{r} / \mathbf{p}\end{array}$ & $\begin{array}{l}\text { ADI-R social } \\
\text { interaction } \mathbf{r} / \mathbf{p}\end{array}$ \\
\hline \multirow{2}{*}{ Medial orbitofrontal } & \multirow{2}{*}{ Right } & Spearman's & $-.73 / .011$ & - & $-.66 / .028$ \\
\hline & & Pearson's & $-.73 / .010$ & - & $-.67 / .022$ \\
\hline \multirow{2}{*}{ Medial orbitofrontal } & \multirow{2}{*}{ Left } & Spearman's & $-.68 / .020$ & - & $-.61 / .048$ \\
\hline & & Pearson's & $-.63 / .039$ & - & - \\
\hline \multirow{2}{*}{ Paracentral } & \multirow{2}{*}{ Left } & Spearman's & - & - & $-.75 / .008$ \\
\hline & & Pearson's & - & - & $-.663 / .026$ \\
\hline \multirow{2}{*}{ Pars opercularis } & \multirow{2}{*}{ Left } & Spearman's & $-.67 / .025$ & - & $-.62 / .042$ \\
\hline & & Pearson's & $-.69 / .020$ & - & - \\
\hline \multirow{2}{*}{ Pars triangularis } & \multirow{2}{*}{ Left } & Spearman's & $-.67 / .025$ & - & $-.73 / .011$ \\
\hline & & Pearson's & - & & - \\
\hline \multirow{2}{*}{ Parietal lobe } & \multirow{2}{*}{ Right } & Spearman's & - & - & $-.73 / .011^{\star}$ \\
\hline & & Pearson's & - & - & - \\
\hline \multirow{2}{*}{ Inferior parietal } & \multirow{2}{*}{ Right } & Spearman's & - & - & $-.73 / .010$ \\
\hline & & Pearson's & - & - & - \\
\hline \multirow{2}{*}{ Precuneus } & \multirow{2}{*}{ Left } & Spearman's & $-.67 / .024$ & - & $-.74 / .009$ \\
\hline & & Pearson's & $-.70 / .016$ & - & $-.68 / .022$ \\
\hline \multirow{2}{*}{ Superior parietal } & \multirow{2}{*}{ Right } & Spearman's & - & $-.61 / .045$ & - \\
\hline & & Pearson's & - & $-.75 / .008$ & - \\
\hline \multirow{2}{*}{ Superior parietal } & \multirow{2}{*}{ Left } & Spearman's & - & $-.69 / .019$ & - \\
\hline & & Pearson's & - & - & - \\
\hline \multirow{2}{*}{ Supramarginal } & \multirow{2}{*}{ Right } & Spearman's & $-.62 / .042$ & - & - \\
\hline & & Pearson's & - & - & - \\
\hline \multirow{2}{*}{ Entorhinal } & \multirow{2}{*}{ Left } & Spearman's & $-.89 /<.001^{*}$ & - & $-.67 / .025$ \\
\hline & & Pearson's & $-.85 / .001$ & - & - \\
\hline \multirow{2}{*}{ Parahippocampal } & \multirow{2}{*}{ Left } & Spearman's & - & - & $-.76 / .007$ \\
\hline & & Pearson's & - & - & - \\
\hline \multirow{2}{*}{ Insula } & \multirow{2}{*}{ Left } & Spearman's & - & - & $-.68 / .020$ \\
\hline & & Pearson's & - & - & - \\
\hline Isthmus cinoulate & Ioft & Spearman's & $-.67 / .025$ & - & $-.73 / .012$ \\
\hline Isthmus cingulate & Left & Pearson's & $-.683 / .02$ & - & - \\
\hline Destorioring & Dight & Spearman's & $-.71 / .015$ & - & $-.68 / .023$ \\
\hline Posterior cingulate & Right & Pearson's & $-.77 / .006$ & - & $-.66 / .026$ \\
\hline Dostorior sing & Ioft & Spearman's & $-.61 / .049$ & - & - \\
\hline Posterior cingulate & Left & Pearson's & - & - & - \\
\hline D t & Dirht & Spearman's & - & - & $-.65 / .030$ \\
\hline Rostral anterior cingulate & Right & \begin{tabular}{|l|} 
Pearson's \\
\end{tabular} & - & - & - \\
\hline
\end{tabular}

Table 3. Spearman's and Pearson's correlations assessing associations between MK and lifetime clinical symptoms as measured by ADI-R. A higher score on the ADI-R indicates increased severity. All tests shown reached trend-level at $p \leq 0.05$, uncorrected. Tests that remain significant after the Benjamini-Hochberg correction for multiple comparisons are indicated by an asterisk $\left(^{*}\right)(\mathrm{q} \leq .05 \mathrm{BH}$ FDR).
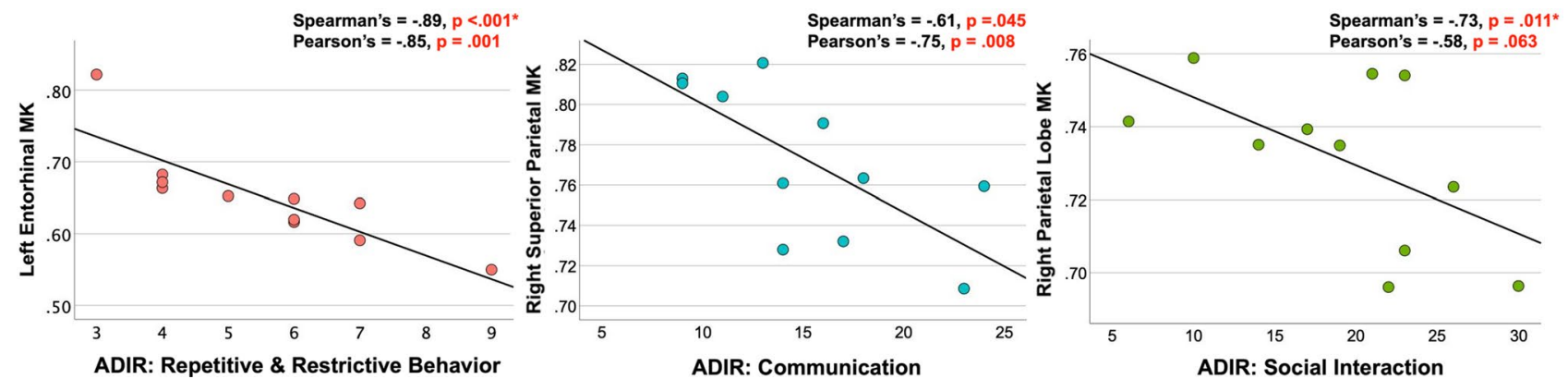

Figure 3. Brain areas with significant associations between performance on the 3 sub-categories of the ADI-R and mean kurtosis in the ASD group. A lower score on the ADI-R indicates less severe symptoms and is associated with increased MK in ASD. The strength of the correlation and the corresponding $p$ value are listed for both Spearman's and Pearson's tests for each graph. 


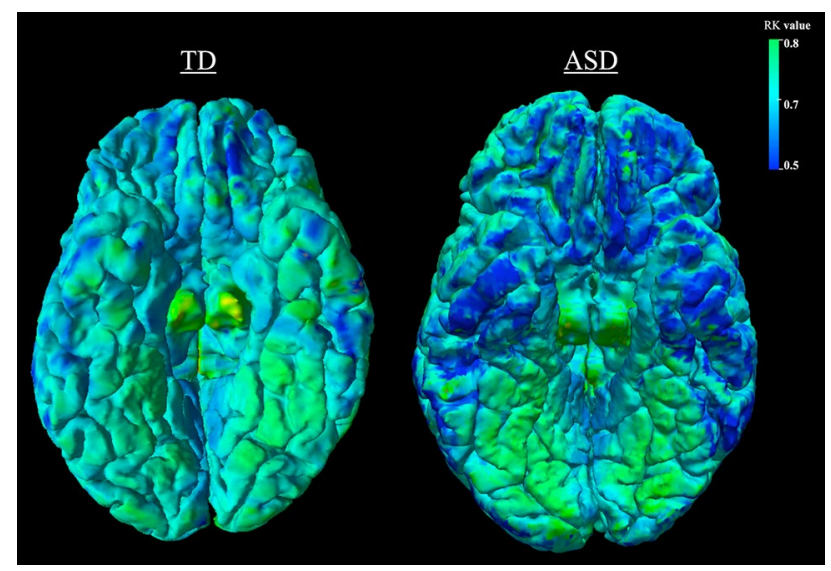

Figure 4. Surface map of radial kurtosis values in a typically developing versus an autism spectrum disorder participant from an inferior view of the temporal, frontal and cingulate areas. RK values are projected from a middle GM cortical layer depth. Dark blue indicates lower values, while bright green indicates higher values.

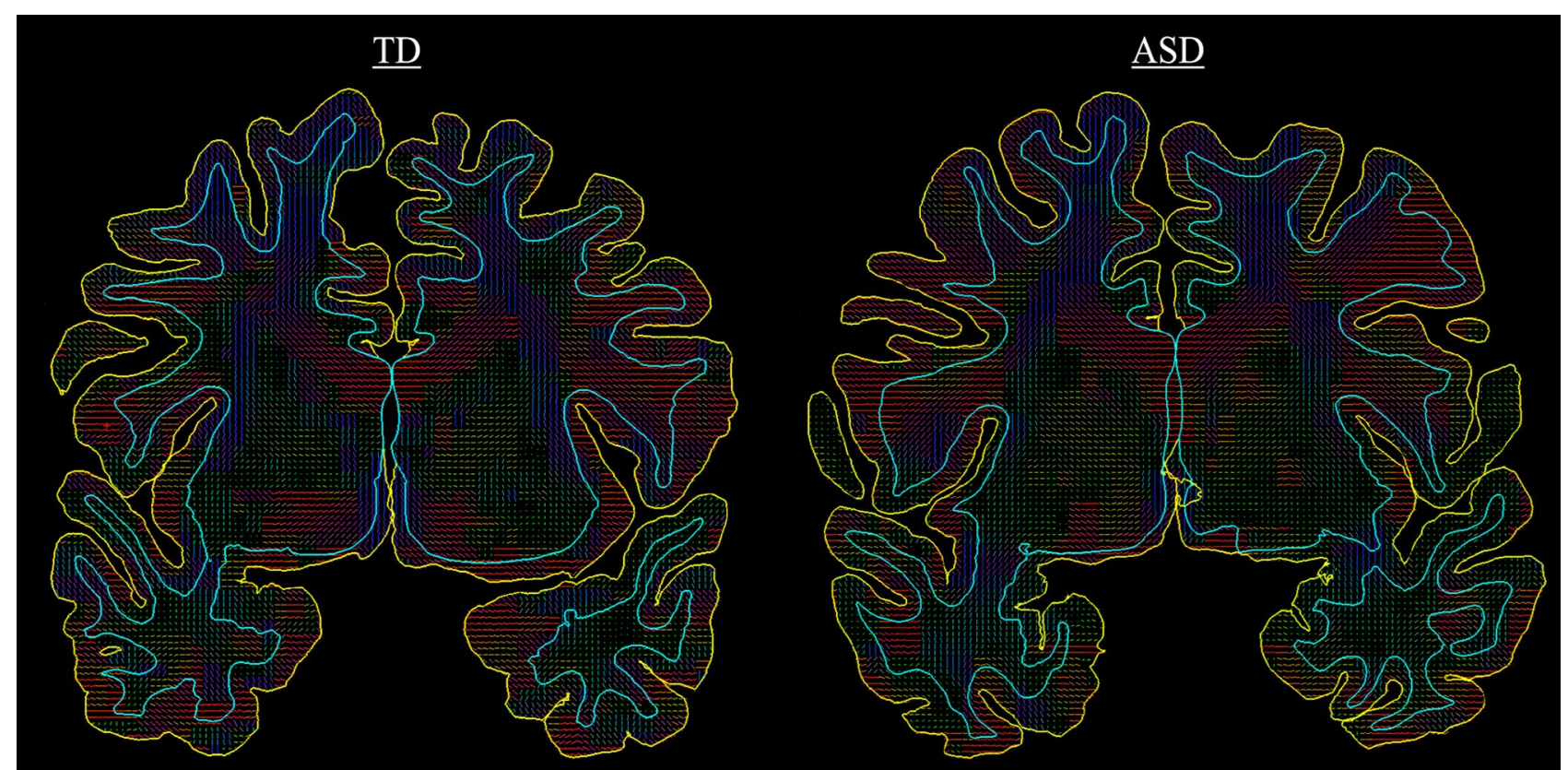

Figure 5. Example of directional diffusion tensors in a mid-brain slice of a typically developing versus autism spectrum disorder participant. Red lines indicate left to right diffusion, blue lines indicate up to down diffusion, and green lines indicate front to back diffusion in the brain. The primary direction of GM diffusion in both brains is largely seen as perpendicular to the cortical mantle (AK), and thus the secondary (radial) diffusion direction is parallel to the cortical mantle (RK).

schizophrenia ${ }^{39,41,73}$. In this study, elevated kurtosis values in ASD, closer to the TD range, appear to be associated with fewer restrictive and repetitive behaviors and better social interaction skills.

Postmortem studies have provided valuable information regarding the underlying GM microstructural pathologies of ASD, most notably reporting increased cell packing ${ }^{4,9}$, decreased cell size and dendritic branching ${ }^{4,10}$ and under-developed minicolumn formation ${ }^{5,13-15}$. However, they are inherently limited by their invasive nature and their findings are difficult to translate into clinical practice and personalized medicine. Advanced dMRI imaging, which is non-invasive and can be acquired in a clinically feasible time frame and setting, may be used to extend and refine these decades-long documented microstructural abnormalities in ASD. Of note, dMRI has mostly been applied to detect in vivo ASD white matter pathologies despite GM showing a larger range of histological abnormalities that may be useful clinical biomarker targets. For example, reduced synaptic density in GM has recently been used as a radiopharmaceutical target in schizophrenia ${ }^{74}$. Kurtosis is an index of microstructural complexity, which in addition to potentially detecting an array of histological changes in ASD it has both successfully revealed the presence of isolated microstructural changes in preclinical models ${ }^{27,75}$ and been superior to conventional macrostructural MRI in differentiating patients in other neurological disorders ${ }^{37,41}$. Therefore, although this pilot study primarily aimed to test the ability of DKI to detect GM microstructural abnormalities 
in ASD and their relationship to symptoms, its results provide support for future larger DKI studies that may better track, predict and treat ASD deficits.

Several limitations of this study need to be considered. First, the sample size was relatively small, and the study had a purely cross-sectional design. In part, our limited sample size stems from our decision to select a relatively homogeneous group by studying males with ASD within a limited age range (18-25 years old) and without intellectual disability (IQ $<80$ ), which we believe strengthened our ability to detect group differences. However, it will be important to replicate these results in larger samples and to examine gray matter in both sexes and across larger age ranges. Second, these data were acquired before the availability of higher resolution diffusion methods that may reveal more accurate diffusion measurements. Third, two different T1-weighted (T1w) MRI protocols were used for the within-subject atlas segmentation and ROI analysis. Paired t-tests showed that different T1w acquisition had no significant effect on the calculation of MK or RK metrics but had some minimal effects on MD metrics and therefore the MD results presented here should be interpreted cautiously (Supplementary Table 3). Finally, given our relatively small sample size, we present both results surviving multiple comparison correction as well as uncorrected results in the tables and supplementary material which may provide the basis for subsequent attempted replications. The study of GM microstructure in ASD is relatively new and we believe the data presented here should encourage further research on the GM substrates of this disorder's clinical manifestations.

In conclusion, we report decreased gray matter kurtosis, primarily arising from altered radial kurtosis, in ASD in the frontal and temporal lobes and in a number of functionally distinct cortical ROIs involved in social and emotional processing. Incorporating in vivo metrics of GM microstructure, such as MK and RK, into models of ASD pathology may be instrumental in better characterizing ASD subtypes and their developmental progressions.

\section{Methods}

Participants. A total of 26 male individuals with a potential ASD diagnosis and 36 age-matched male TD participants were recruited by advertisement within the community and through autism organizations in the New York City metropolitan area. Diagnoses were confirmed by administering the Autism Diagnostic Observation Schedule (ADOS) ${ }^{76}$ to all ASD participants under the supervision of a certified licensed clinical psychologist. Additionally the ADI- ${ }^{72}$ was administered to the ASD parents who participated in the study. TD participants who reported no personal or family history of ASD or other psychiatric disorders were retained in the study. Participants were excluded for a history of head trauma, organic brain disorder, IQ $<80$, and MRI contraindications. The Weschler Adult Intelligence scale (WAIS-III) ${ }^{77}$ was administered to obtain Full Scale intellectual quotient (FSIQ) scores and confirm the lack of intellectual disability. Handedness was obtained using the Chapman and Chapman handedness questionnaire ${ }^{78}$. The study was approved by the institutional review board at the NYU School of Medicine and performed in accordance with all National Institute of Mental Health (NIMH) and NYU School of Medicine relevant guidelines and regulations. For this study, 17 ASD patients and their parents provided informed consent. For another 9 ASD participants, for whom parents were not available for participation, competence to provide consent was assessed at the beginning of the visit by trained personnel. All these study participants were adults deemed able to provide informed consent and provided informed consent before participation according to our institutional rules.

Subjects whose imaging data displayed significant motion artifacts and for whom repeated data acquisition was not successful were not included in the study. A neuroradiologist examined all scans for gross brain abnormalities and to exclude any subjects suspected of an organic brain disorder.

To match the two groups on IQ, TD subjects underwent first only a review of medical history and the IQ assessment. As new ASD participants were added to the study, TD participants were invited for imaging if they had IQ values similar to the recruited ASD participants. Twelve TD participants were either no longer available to participate for imaging by the time they were invited or had not matched any of the ASD participants and were thus dropped from the study. In addition, seven other TD participants met exclusionary criteria, which included a previous or current diagnosis of attention deficit hyperactivity disorder (2 participants), leukemia ( 1 participant), or MRI findings (4 participants). Among the potential ASD participants, two were excluded due to IQ $<80$, three since they did not meet the threshold criteria for an ASD diagnosis, and one due to the presence of MRI contraindications. Two additional ASD participants requested to terminate the MRI procedure before any meaningful data could be acquired. Finally, two additional ASD data sets were not used in this study due to poor image quality. The remaining data sets (17 TD and 16 ASD participants) were included in the analyses (Table 1). Among the included ASD participants, 11 had participating parents that underwent the ADI-R.

Magnetic resonance image acquisition. All MRI data were acquired on a 3T Trio MRI (Siemens Medical Solutions, Erlangen, Germany). Images were acquired using a body coil for transmission and a 12-channel array coil for reception. Diffusion imaging data were acquired using a twice-refocused diffusion-weighted echo planar imaging (EPI) sequence with a GRAPPA parallel imaging factor 2, and 24 reference lines. Between 55 and 60 slices were acquired using an isotropic voxel size of $2.3 \times 2.3 \times 2.3 \mathrm{~mm}^{3}$, TR=8100 ms, and TE=97 ms. Diffusion weighted imaging data were acquired for two $b$ values $\left(b=1000\right.$ and $\left.2000 \mathrm{~s} / \mathrm{mm}^{2}\right)$ with 12 non-collinear encoding directions acquired for $b=1000 \mathrm{~s} / \mathrm{mm}^{2}$, and 42 non-collinear encoding directions and for $b=2000 \mathrm{~s} /$ $\mathrm{mm}^{2}$. Ten non-weighted diffusion images $\left(b=0 \mathrm{~s} / \mathrm{mm}^{2}\right)$ were also collected. Diffusion data acquisition was repeated twice. To correct for image distortions from B0 field inhomogeneities phase and magnitude field map images were acquired coplanar to the diffusion acquisition using the Siemens product sequence with echo-times of $8 \mathrm{~ms}$ and $10.46 \mathrm{~ms}$.

In addition to the diffusion data, T1w images were acquired using a magnetization prepared rapid gradient-echo (MPRAGE) sequence and used in the atlas registration procedure for ROI analyses, and additionally for clinical evaluation of gross brain abnormalities in each subject. Two T1w protocols were used: a) an axial 
MPRAGE with a $192 \times 256 \times 188$ matrix and a $1 \mathrm{~mm}^{3}$ isotropic voxel size was collected for 8 participants $(2$ ASD, $6 \mathrm{TD}$ ), and b) a MPRAGE with a $160 \times 240 \times 256$ matrix and a $1 \times 1 \times 1.2 \mathrm{~mm}$ voxel size was collected for 25 participants (14 ASD, 11 TD). Eleven participants ( 9 ASD and 2 TD) had both T1w acquisitions with the rest of the participants having only one MPRAGE image.

Image processing. Data were preprocessed and images were corrected for motion and distortions from eddy currents along with magnetic field inhomogeneities using in-house developed code in Matlab (Mathworks, Natick, Massachusetts), Interactive Data Language (IDL, Exelis Visual Information Solutions, Boulder, Colorado), and FMRIB Software Library (FSL4.1,http://www.fmrib.ox.ac.uk/fsl) ${ }^{79}$. Data pre-processing steps included: (1) correction of B0 field inhomogeneities using the field map and FSL fugue and prelude functions; (2) image smoothing with a three-dimensional Gaussian filter with $\sigma=1.2 \mathrm{~mm}$; (3) visual inspection of images for signal dropouts, blurring from movement and removal of artefactual images, and; (4) adjustment of the encoding gradients' matrix for rotations during the motion correction step. After image correction, diffusion and kurtosis tensors were calculated as previously described in the field ${ }^{80}$ and employed to calculate threedimensional maps of MK and MD. Additionally, AK and RK maps were obtained to test for directional differences in kurtosis microstructural properties.

FreeSurfer (http://surfer.nmr.mgh.harvard.edu/; version 6.0) was used to construct the cortical surface of each participant based on the high-resolution T1w image. The FreeSurfer pipeline for surface construction, processed the images by segmentation of tissue types, tessellation of the grey/white matter junction, inflation of the folded surface and parcellation by the Desikan-Killiany atlas ${ }^{81}$. Each subject-specific cortically-labeled volume was then warped into dMRI space using FreeSurfer's rigid-body transformation ${ }^{81}$. Mean diffusion metrics were obtained for each of the four regional cortical lobes and 68 cerebral cortex GM ROIs for each subject and used to conduct group comparisons.

To test if the use of two T1w acquisitions had an effect on dMRI metric values shown to be significantly different between groups (MK, RK, and MD) we conducted paired t-tests on data from 11 participants (9 ASD, 2 TD) that had both T1w acquisitions. For each of the 11 participants, image processing and registration to diffusion space was done separately for both T1w acquisitions and dMRI metrics were subsequently compared in the 68 ROIs delineated by the Desikan-Killiany atlas ${ }^{82}$. We found that T1w acquisition had no significant effect on RK or MK metrics ( $p>0.05$ for all comparisons) and minimal effect on MD metrics with no overlap between the ROIs affected by the T1W protocol and those shown to have significantly different MD in between-group comparisons (Supplementary Table 3).

Statistical analyses. Primary analyses used analysis of covariance (ANCOVA) tests controlling for age to first compare diffusion metrics (MK, AK, RK and MD) across the four anatomically defined cortical lobes (frontal, parietal, temporal, and occipital), and then secondarily across the 68 cortical sub-lobar GM ROIs delineated by the Desikan-Killiany atlas ${ }^{82}$ using SPSS 20.0 (IBM, Armonk, NY). Although the two groups did not statistically differ in demographics (Table 1), DKI metrics have been shown to be sensitive to age ${ }^{24,34-36}$ and therefore age was included as a covariate in these analyses. Cohen's d effect size was calculated to compare group means for each ROI analysis.

Pearson's and Spearman's correlations were used to explore the relationships between diffusion metrics that were found to show significant between-group differences (MK, RK, and MD) and ADI-R domain scores in ASD.

To account for multiple comparisons across the four cortical lobes and 68 cortical regions, we employed the Benjamini-Hochberg $(\mathrm{BH})$ procedure ${ }^{52}$ to control the False Discovery Rate at $5 \%$. Multiple comparison correction was applied first to (1) the 8 bilateral GM lobes, and then to (2) the 68 sub-cortical GM ROIs delineated by the Desikan-Killiany atlas. The Benjamini-Hochberg multiple comparison correction was performed on a single $\mathrm{dMRI}$ variable (MK, RK, $\mathrm{AK}$ and $\mathrm{MD}$ ) at a time. Individual results were considered significant at $\mathrm{BH} \mathrm{q} \leq 0.05$. Differences at $p \leq 0.05$, uncorrected were considered to only reach trend-level and are listed in the tables and in the supplementary material.

Received: 4 May 2020; Accepted: 29 October 2020

Published online: 08 December 2020

\section{References}

1. American Psychiatric Association. DSM-5 Diagnostic Classification. Diagnostic and Statistical Manual of Mental Disorders (5th ed.) (2013).

2. Blumberg, S. J. et al. Changes in prevalence of parent-reported autism spectrum disorder in school-aged U.S. children: 2007 to 2011-2012. Natl. Health Stat. Report. 20, 1-11 (2013).

3. Dementieva, Y. A. et al. Accelerated head growth in early development of individuals with autism. Pediatr. Neurol. 32, 102-108 (2005).

4. Bailey, A. et al. A clinicopathological study of autism. Brain 121, 889-905 (1998).

5. Casanova, M. F. et al. Minicolumnar abnormalities in autism. Acta Neuropathol. 112, 287-303 (2006).

6. Di Martino, A. et al. The autism brain imaging data exchange: Towards a large-scale evaluation of the intrinsic brain architecture in autism. Mol. Psychiatry 19, 659-667 (2014).

7. Travers, B. G. et al. Diffusion tensor imaging in autism spectrum disorder: A review. Autism Res. 5, 289-313 (2012).

8. Ameis, S. H. \& Catani, M. Altered white matter connectivity as a neural substrate for social impairment in autism spectrum disorder. Cortex 62, 158-181 (2015).

9. Courchesne, E. et al. Neuron number and size in prefrontal cortex of children with autism. J. Am. Med. Assoc. 306, 2001-2010 (2011).

10. Kemper, T. L. \& Bauman, M. Neuropathology of infantile autism. J. Neuropathol. Exp. Neurol. 57, 645-652 (1998). 
11. Raymond, G. V., Bauman, M. L. \& Kemper, T. L. Hippocampus in autism: a Golgi analysis. Acta Neuropathol. 91, 117-119 (1995).

12. Vargas, D. L., Nascimbene, C., Krishnan, C., Zimmerman, A. W. \& Pardo, C. A. Neuroglial activation and neuroinflammation in the brain of patients with autism. Ann. Neurol. 57, 67-81 (2005).

13. Casanova, M. F. The neuropathology of autism. Mol. Basis Autism 17, 422-433 (2015).

14. Casanova, M. F., Switala, E. A., Trippe, J. \& Fitzgerald, M. Comparative minicolumnar morphometry of three distinguished scientists. Autism 11, 557-5569 (2007).

15. Buxhoeveden, D. P. et al. Reduced minicolumns in the frontal cortex of patients with autism. Neuropathol. Appl. Neurobiol. 32, 483-491 (2006).

16. Casanova, M. F., Buxhoeveden, D. P. \& Brown, C. Clinical and macroscopic correlates of minicolumnar pathology in autism. J. Child Neurol. 17, 692-695 (2003).

17. Palmen, S. J. M. C., Van Engeland, H., Hof, P. R. \& Schmitz, C. Neuropathological findings in autism. Brain 127, 2572-2583 (2004).

18. Aung, W. Y., Mar, S. \& Benzinger, T. L. Diffusion tensor MRI as a biomarker in axonal and myelin damage. Imaging Med. 5, 427-440 (2013).

19. Wang, Y. et al. Differentiation and quantification of inflammation, demyelination and axon injury or loss in multiple sclerosis. Brain 138, 1223-1238 (2015).

20. Song, S. K. et al. Dysmyelination revealed through MRI as increased radial (but unchanged axial) diffusion of water. Neuroimage 17, 1429-1436 (2002).

21. Lazar, M., Miles, L. M., Babb, J. S. \& Donaldson, J. B. Axonal deficits in young adults with high functioning autism and their impact on processing speed. NeuroImage Clin. 7, 417-425 (2014).

22. Aggarwal, M., Nauen, D. W., Troncoso, J. C. \& Mori, S. Probing region-specific microstructure of human cortical areas using high angular and spatial resolution diffusion MRI. Neuroimage 105, 198-207 (2015).

23. Wu, E. X. \& Cheung, M. M. MR diffusion kurtosis imaging for neural tissue characterization. NMR Biomed. 23, 836-848 (2010).

24. Helpern, J. A. et al. Preliminary evidence of altered gray and white matter microstructural development in the frontal lobe of adolescents with attention-deficit hyperactivity disorder: A diffusional kurtosis imaging study. J. Magn. Reson. Imaging 33, 17-23 (2011).

25. Jensen, J. H., Helpern, J. A., Ramani, A., Lu, H. \& Kaczynski, K. Diffusional kurtosis imaging: The quantification of non-Gaussian water diffusion by means of magnetic resonance imaging. Magn. Reson. Med. 53, 1432-1440 (2005).

26. Steven, A. J., Zhuo, J. \& Melhem, E. R. Diffusion kurtosis imaging: An emerging technique for evaluating the microstructural environment of the brain. Am. J. Roentgenol. 202, 26-33 (2014).

27. Vanhoutte, G. et al. Diffusion kurtosis imaging to detect amyloidosis in an APP/PS1 mouse model for Alzheimer's disease. Magn. Reson. Med. 69, 1115-1121 (2013).

28. Zhuo, J. et al. Diffusion kurtosis as an in vivo imaging marker for reactive astrogliosis in traumatic brain injury. Neuroimage 59, 467-477 (2012).

29. Blockx, I. et al. Identification and characterization of Huntington related pathology: An in vivo DKI imaging study. Neuroimage 63, 653-662 (2012).

30. Palacios, R. D. Y., Verhoye, M., Henningsen, K., Wiborg, O. \& Van Der Linden, A. Diffusion kurtosis imaging and high-resolution mri demonstrate structural aberrations of caudate putamen and amygdala after chronic mild stress. PLoS ONE 9, 1-8 (2014).

31. Wang, M. L. et al. Diffusion kurtosis imaging characterizes brain microstructural changes associated with cognitive impairment in a rat model of chronic traumatic brain injury. Neuroscience 392, 180-189 (2018).

32. Nie, X. et al. Evidence of altered age-related brain cytoarchitecture in mouse models of down syndrome: A diffusional kurtosis imaging study. Magn. Reson. Imaging 33, 437-447 (2015).

33. McNab, J. A. et al. Surface based analysis of diffusion orientation for identifying architectonic domains in the in vivo human cortex. Neuroimage 69, 87-100 (2013).

34. Ota, M. et al. Whole brain analyses of age-related microstructural changes quantified using different diffusional magnetic resonance imaging methods. Jpn. J. Radiol. 35, 584-589 (2017).

35. Das, S. K., Wang, J. L., Bing, L., Bhetuwal, A. \& Yang, H. F. Regional values of diffusional kurtosis estimates in the healthy brain during normal aging. Clin. Neuroradiol. 27, 283-298 (2017).

36. Falangola, M. F. et al. Age-related non-Gaussian diffusion patterns in the prefrontal brain. J. Magn. Reson. Imaging 28, 1345-1350 (2008).

37. Zhang, G. et al. Diffusion kurtosis imaging of substantia Nigra is a sensitive method for early diagnosis and disease evaluation in Parkinson's disease. Parkinsons Dis. 2015, 1-5 (2015).

38. Zhang, Y. et al. Microstructural abnormalities in gray matter of patients with postherpetic Neuralgia: A diffusional kurtosis imaging study. Pain Phys. 19, 601-611 (2016).

39. Bester, M. et al. Non-Gaussian diffusion MRI of gray matter is associated with cognitive impairment in multiple sclerosis. Mult. Scler. 21, 935-944 (2015).

40. Stokum, J. A. et al. A longitudinal evaluation of diffusion kurtosis imaging in patients with mild traumatic brain injury. Brain Inj. 29, 47-57 (2015).

41. McKenna, F. F., Miles, L., Babb, J. S., Goff, D. C. \& Lazar, M. Diffusion kurtosis imaging of gray matter in schizophrenia. Cortex 121, 201-224 (2019)

42. Falangola, M. F. et al. Non-Gaussian diffusion MRI assessment of brain microstructure in mild cognitive impairment and Alzheimer's disease. Magn. Reson. Imaging 31, 840-846 (2013).

43. Adisetiyo, V. et al. Attention-deficit/hyperactivity disorder without comorbidity is associated with distinct atypical patterns of cerebral microstructural development. Hum. Brain Mapp. 35, 2148-2162 (2014).

44. Chen, X. R., Zeng, J. Y., Shen, Z. W., Kong, L. M. \& Zheng, W. Bin. Diffusion kurtosis imaging detects microstructural changes in the brain after acute alcohol intoxication in rats. Biomed Res. Int. 2017, 1-6 (2017).

45. Braeckman, K. et al. Dynamic changes in hippocampal diffusion and kurtosis metrics following experimental mTBI correlate with glial reactivity. NeuroImage Clin. 21, 1-12 (2019).

46. Cheung, M. M. et al. Does diffusion kurtosis imaging lead to better neural tissue characterization? A rodent brain maturation study. Neuroimage 45, 386-392 (2009).

47. Courchesne, E. \& Pierce, K. Brain overgrowth in autism during a critical time in development: Implications for frontal pyramidal neuron and interneuron development and connectivity. Int. J. Dev. Neurosci. 23, 153-170 (2005).

48. Groen, W. B., Buitelaar, J. K., van der Gaag, R. J. \& Zwiers, M. P. Pervasive microstructural abnormalities in autism: A DTI study. J. Psychiatry Neurosci. 36, 32-40 (2011).

49. Carper, R. A., Treiber, J. M., White, N. S., Kohli, J. S. \& Müller, R. A. Restriction spectrum imaging as a potential measure of cortical neurite density in autism. Front. Neurosci. 10, 610-619 (2017).

50. Yasuno, F. et al. Microstructural anomalies evaluated by neurite orientation dispersion and density imaging are related to deficits in facial emotional recognition via perceptual-binding difficulties in autism spectrum disorder. Autism Res. 13, 729-740 (2020).

51. Yang, A. W. et al. Effect of cerebral spinal fluid suppression for diffusional kurtosis imaging. J. Magn. Reson. Imaging 37, 365-371 (2013).

52. Hochberg, B. controlling the false discovery rate: A practical and powerful approach to multiple testing. J. R. Stat. Soc. 57, 289-300 (1995). 
53. Irie, R. et al. The relationship between neurite density measured with confocal microscopy in a cleared mouse brain and metrics obtained from diffusion tensor and diffusion kurtosis imaging. Magn. Reson. Med. Sci. 17, 138-144 (2017).

54. Kleinnijenhuis, M. et al. Detailed laminar characteristics of the human neocortex revealed by NODDI and histology. in Organization for Human Brain Mapping (2013).

55. Budde, M. D., Janes, L., Gold, E., Turtzo, L. C. \& Frank, J. A. The contribution of gliosis to diffusion tensor anisotropy and tractography following traumatic brain injury: Validation in the rat using Fourier analysis of stained tissue sections. Brain 134, 2248-2260 (2011).

56. Jespersen, S. N., Leigland, L. A., Cornea, A. \& Kroenke, C. D. Determination of axonal and dendritic orientation distributions within the developing cerebral cortex by diffusion tensor imaging. IEEE Trans. Med. Imaging 31, 16-32 (2012).

57. Cottaar, M. et al. A gyral coordinate system predictive of fibre orientations. Neuroimage 176, 417-430 (2018).

58. Gulban, O. F. et al. Cortical fibers orientation mapping using in-vivo whole brain 7 T diffusion MRI. Neuroimage 178, 104-118 (2018).

59. Edwards, L. J., Kirilina, E., Mohammadi, S. \& Weiskopf, N. Microstructural imaging of human neocortex in vivo. Neuroimage 182, 184-206 (2018).

60. Courchesne, E. \& Pierce, K. Why the frontal cortex in autism might be talking only to itself: Local over-connectivity but longdistance disconnection. Curr. Opin. Neurobiol. 15, 225-230 (2005).

61. Gervais, H. et al. Abnormal cortical voice processing in autism. Nat. Neurosci. 7, 801-802 (2004).

62. Just, M. A., Cherkassky, V. L., Keller, T. A. \& Minshew, N. J. Cortical activation and synchronization during sentence comprehension in high-functioning autism: Evidence of underconnectivity. Brain 127, 1811-1821 (2004).

63. Kemper, T. L. \& Bauman, M. L. The contribution of neuropathologic studies to the understanding of autism. Neurol. Clin. 11, 175-187 (1993).

64. Carper, R. A. \& Courchesne, E. Localized enlargement of the frontal cortex in early autism. Biol. Psychiatry 57, 126-133 (2005).

65. Amaral, D. G., Schumann, C. M. \& Nordahl, C. W. Neuroanatomy of autism. Trends Neurosci. 31, 137-145 (2008).

66. Blakemore, S. J. The social brain in adolescence. Nat. Rev. Neurosci. 105, 111-116 (2008).

67. Velikonja, T., Fett, A.-K. \& Velthorst, E. Patterns of nonsocial and social cognitive functioning in adults with autism spectrum disorder. JAMA Psychiatry 10029, 135-151 (2019).

68. Golarai, G., Grill-Spector, K. \& Reiss, A. L. Autism and the development of face processing. Clin. Neurosci. Res. 6, 145-160 (2006).

69. Castelli, F. Autism, Asperger syndrome and brain mechanisms for the attribution of mental states to animated shapes. Brain 125, $1839-1849$ (2002).

70. Crowley, M. J. et al. Brain responses to biological motion predict treatment outcome in young children with autism. Transl. Psychiatry 6, 948-949 (2016).

71. Lombardo, M. V. et al. Atypical neural self-representation in autism. Brain 133, 611-624 (2010).

72. Lord, C., Rutter, M. \& Le Couteur, A. Autism diagnostic interview-revised: A revised version of a diagnostic interview for caregivers of individuals with possible pervasive developmental disorders. J. Autism Dev. Disord. 24, 659-685 (1994).

73. Grossman, E. J. et al. Cognitive impairment in mild traumatic brain injury: A longitudinal diffusional kurtosis and perfusion imaging study. Am. J. Neuroradiol. 34, 951-957 (2013).

74. Nazeri, A., Schifani, C., Anderson, J. A. E., Ameis, S. H. \& Voineskos, A. N. In vivo imaging of gray matter microstructure in major psychiatric disorders: Opportunities for clinical translation. Biol. Psychiatry Cogn. Neurosci. Neuroimaging 5, 855-864 (2020)

75. Khairnar, A. et al. Early and progressive microstructural brain changes in mice overexpressing human $\alpha$-Synuclein detected by diffusion kurtosis imaging. Brain. Behav. Immun. 61, 197-208 (2017).

76. Lord, C. et al. The Autism Diagnostic Observation Schedule-Generic: A standard measure of social and communication deficits associated with the spectrum of autism. J. Autism Dev. Disord. 30, 205-223 (2000).

77. Wechsler, D. Wechsler Adult Intelligence Scale - Fourth Edition. Pearson (Pearson, 2008). https://doi.org/10.1037/t15169-000

78. Chapman, L. J. \& Chapman, J. P. The measurement of handedness. Brain Cogn. 6, 175-183 (1987).

79. Jenkinson, M., Beckmann, C. F., Behrens, T. E., Woolrich, M. W. \& Smith, S. M. Fsl. Neuroimage 62, 782-790 (2012).

80. Tabesh, A., Jensen, J. H., Ardekani, B. A. \& Helpern, J. A. Estimation of tensors and tensor-derived measures in diffusional kurtosis imaging. Magn. Reson. Med. 65, 823-836 (2011).

81. Fischl, B. \& Dale, A. M. Measuring the thickness of the human cerebral cortex from magnetic resonance images. Proc. Natl. Acad. Sci. 97, 11050-11055 (2000).

82. Desikan, R. S. et al. An automated labeling system for subdividing the human cerebral cortex on MRI scans into gyral based regions of interest. Neuroimage 31, 968-980 (2006).

\section{Acknowledgements}

We greatly thank all our participants for their help with this study and the Global and Regional Asperger Syndrome Partnership (GRASP), Interactive Autism Network (IAN), Asperger Syndrome and High Functioning Autism Association (AHANY), and ResearchMatch for their support in advertising the study and recruiting participants. We also thank Kelly Anne McGorty, William Fazio, David Mossa and Kamil Banibaker for their assistance with acquiring the MR data. This study was funded in part by the National Institutes of Mental Health through NIMH R03-MH076180 award.

\section{Author contributions}

F.M. contributed to data processing, statistical analysis, interpretation and manuscript writing. L.M. and J.D. carried out diagnostic confirmation and cognitive assessments. F.X.C. contributed to data interpretation and manuscript writing. M.L. contributed to the conception, design, data acquisition, analysis, interpretation and manuscript writing. All authors revised and approved the final manuscript.

\section{Competing interests}

The authors declare no competing interests.

\section{Additional information}

Supplementary Information The online version contains supplementary material available at https://doi. org/10.1038/s41598-020-78486-w.

Correspondence and requests for materials should be addressed to F.M.

Reprints and permissions information is available at www.nature.com/reprints. 
Publisher's note Springer Nature remains neutral with regard to jurisdictional claims in published maps and institutional affiliations.

(c) (1) Open Access This article is licensed under a Creative Commons Attribution 4.0 International License, which permits use, sharing, adaptation, distribution and reproduction in any medium or format, as long as you give appropriate credit to the original author(s) and the source, provide a link to the Creative Commons licence, and indicate if changes were made. The images or other third party material in this article are included in the article's Creative Commons licence, unless indicated otherwise in a credit line to the material. If material is not included in the article's Creative Commons licence and your intended use is not permitted by statutory regulation or exceeds the permitted use, you will need to obtain permission directly from the copyright holder. To view a copy of this licence, visit http://creativecommons.org/licenses/by/4.0/.

(c) The Author(s) 2020 